76 FOUNDATION DOCTORS AS LEADERS OF FIRST AID TEACHING AND HEALTH PROMOTION IN UK SECONDARY SCHOOLS THROUGH \#HEALTHINSCHOOLS: A NATIONAL PROGRAMME ENDORSED BY THE UKFPO

\begin{abstract}
1,2Marina Soltan, 1,2 Liz Sapey, ${ }^{3}$ Colin Melville, ${ }^{4,5,6}$ Tony Choules, ${ }^{6}$ Carrie MacEwen, ${ }^{1}$ Peter Nightingale, ${ }^{1,2}$ David Thickett, ${ }^{5,7}$ Clare van Hamel, ${ }^{8}$ Tony Young. ${ }^{1}$ University of Birmingham, UK; ${ }^{2}$ University Hospitals of Birmingham, Queen Elizabeth Hospital, UK; ${ }^{3}$ General Medical Council, UK; ${ }^{4}$ University Hospital of Derby and Burton, UK; ${ }^{5}$ Health Education UK; ${ }^{6}$ Academy of Medical Royal Colleges, UK; ${ }^{7}$ UKFPO, UK; ${ }^{8}$ NHS UK
\end{abstract}

\subsection{6/leader-2019-FMLM.76}

Background The present study, \#HealthinSchools, explores health professionals in training as leaders of first aid teaching and health promotion in UK secondary schools. First aid teaching in schools is associated with improved cardiac arrest outcomes and was mandated by the UK government in January 2019. Diet, sleep hygiene, smoking cessation, alcohol moderation and 20 minutes of daily exercise have been shown to prevent cardio-respiratory disease and alleviate depression.

Aims This study aims to: A) Identify an evidence-based approach to lead first aid teaching in UK schools, B) Promote behaviour change to prevent modifiable risk factors and comorbidities, C) Up-skill foundation doctors with leadership skills, D) Drive a sustainable NHS workforce which represents the community it serves.

Methods First aid teaching was delivered by 51 foundation doctors to 3251 UK secondary school children in 17 schools in regions of varying deprivation. Teaching sessions lasted one hour and were structured to include an: introduction, interactive face to face masterclass style demonstration, opportunity for pupils to practice on mannequins the psychomotor skills involved, including CPR, whilst being observed and a plenary. Pre and post-course questionnaires and focus groups were carried out among pupils and foundation doctors.

Results Results identified a significant improvement in trainee healthcare professionals' perceived leadership skills and pupils' knowledge and confidence of first aid skills. A statistically significant correlation was noted between schools in more deprived regions and increased pupil aspirations to: 1) incorporate 20 minutes of exercise daily and 2) apply for NHS careers.

Conclusions \#HealthinSchools establishes healthcare professionals as leaders of effective first-aid teaching in UK secondary schools with potential benefits for widening participation to NHS careers and promoting healthy behaviour changes to reduce cardio-respiratory disease morbidity.

\section{USE OF ANTIPLATELET AND STATIN THERAPY IN PATIENTS WITH PERIPHERAL ARTERIAL DISEASE: A CLOSED LOOP AUDIT}

Ammar Ahmed, Kiran Patel, Oliver Lyons, Shiva Dindyal. Vascular Surgery, Basildon University Hospital, UK

\subsection{6/leader-2019-FMLM.77}

Introduction Peripheral arterial disease (PAD) is increasing in prevalence worldwide and is associated with significant risk of cardiovascular complications. NICE guidelines advocate combination therapy with clopidogrel $75 \mathrm{mg}$ and atorvastatin 80 $\mathrm{mg}$ as first line for secondary prevention of PAD. We conducted a closed-loop audit to investigate whether patients with PAD admitted under the vascular surgical team at Basildon University Hospital (BTUH) were appropriately prescribed antiplatelet and statin therapy in accordance with NICE guidelines.

Methods An initial audit was performed investigating which antiplatelets and statins vascular surgical patients were prescribed during admission to BTUH. Data was collected over a 2-week period in December 2018 and presented at the local surgical audit meeting. Additionally, results were presented as an educational intervention at departmental teaching sessions aimed at junior doctors. A re-audit was conducted in April 2019 to evaluate the effectiveness of interventional strategies.

Results A total of 12 vascular patients were included with a mean age of 68 years. Co-morbidities included diabetes, CKD, hypertension, heart failure. Marked improvement was observed in both antiplatelet and statin prescribing following intervention. All patients received antiplatelets, with $50 \%$ being prescribed clopidogrel $75 \mathrm{mg}$ alone, an increase from $13 \%$ observed in the initial audit. Further, all patients received statins, with $66.7 \%$ of patients being prescribed atorvastatin 80 $\mathrm{mg}$, an increase from $26.7 \%$ seen in our initial audit.

Conclusion Patients with PAD should be commenced on clopidogrel $75 \mathrm{mg}$ daily and atorvastatin $80 \mathrm{mg}$ daily. Our results demonstrate that significant improvements were made with educational intervention and effective multidisciplinary cooperation. Our work may be replicated in different hospital trusts nationally with the aim of improving compliance with NICE guidance on secondary prevention of PAD.

\section{LEADING TRANSFORMATION IN A REGIONAL NHS TERTIARY REFERRAL SERVICE (THE LATERAL SKULL BASE SERVICE IN LEEDS)}

Sanjay Verma, Kenan Deniz. Leeds Teaching Hospitals NHS Trust, UK

\subsection{6/leader-2019-FMLM.78}

Leeds provides a regional tertiary referral service for vestibular schwannoma (also known as acoustic neuroma) and other lateral skull base pathologies, receiving referrals from across West Yorkshire. Such were the increasing demands on the service, it was struggling to keep pace with the volume of new referrals and follow up of existing patients, leading to unacceptably high waiting times for clinic appointments and results.

In March 2018 Mr Sanjay Verma assumed the position of Clinical Lead for the Lateral Skull Base service and led it through National Peer review. As a consequence of this several areas for improvement were identified. Mr Verma, and his neurosurgical Consultant colleague, Mr Kenan Deniz, devised an Action Plan, aimed at transforming the service, both to bring it in line with national guidelines and also to improve the quality of the service delivered to patients.

Firstly, a system of virtual follow up was introduced for patients being managed conservatively with serial MRI scanning. This has significantly improved the patient experience by obviating the needs for many patients to travel long distances for follow up appointments.

Secondly, Mr Verma and Mr Deniz engaged with Public Health physicians in a Better Value Healthcare project to determine the specific needs of the Lateral Skull base service, taking into account the perspectives of clinicians and patients. 